\title{
Efficient excitation of self-collimated beams and single Bloch modes in planar photonic crystals
}

\author{
Jeremy Witzens and Axel Scherer \\ Department of Electrical Engineering, MC 136-93, California Institute of Technology, Pasadena, California 91125
}

Received October 7, 2002; revised manuscript received December 13, 2002; accepted January 17, 2003

\begin{abstract}
Using finite-difference time-domain calculations, we investigate out-of-plane coupling between a square-lattice planar photonic crystal and a conventional waveguide located above the photonic crystal. We couple a waveguide oriented in the $\Gamma X$ direction to a photonic crystal mode in the second band and show that anticrossing takes place. In this way, a self-collimated beam is launched in the planar photonic crystal, with full power transfer. Furthermore, we investigate the coupling between a waveguide oriented in the $\Gamma M$ direction and a photonic crystal and show that single photonic crystal modes can be selectively excited. (C) 2003 Optical Society of America
\end{abstract}

OCIS code: 130.1750 .

\section{INTRODUCTION}

Photonic crystals ${ }^{1}$ have attracted a lot of attention owing to their ability to control the flow of light on very small length scales. One class of photonic crystals, planar photonic crystals ${ }^{2}$ (PPCs), represents particularly promising structures for integrated optics, since their planar fabrication allows the use of conventional microelectronics patterning techniques. A PPC is an optically thin dielectric slab perforated with a two-dimensional (2D) lattice of holes. Light is confined within the slab in the vertical direction through total internal reflection and in the lateral direction by distributed Bragg reflection, from the 2D lattice of holes. ${ }^{3,4}$ By creating different types of defect in a photonic crystal, it is possible to make lasers ${ }^{5}$ and waveguides, ${ }^{6}$ as well as coupled photonic devices. ${ }^{7,8}$ There has been an ongoing effort to develop efficient methods to couple light in and out of photonic crystal structures. $^{9,10}$ In particular, there have been several investigations on the coupling to free space from photonic crystal modes ${ }^{11}$ and from photonic crystal waveguide modes. ${ }^{12}$ Furthermore, there have been several investigations on how to combine photonic-crystal-based devices with more conventional integrated optics. ${ }^{13,14}$

More recently there has been a growing interest in the intrinsic dispersion properties of photonic crystals, such as self-collimation ${ }^{15}$ and the superprism effect. ${ }^{16}$ Selfcollimation arises when the equifrequency contours of photonic crystals have approximately flat sides. Because the group velocity is perpendicular to the equifrequency contours, light with a broad range of $k$ vectors that would normally diffract remains collimated in such a photonic crystal. The difficulty in coupling to photonic crystal Bloch modes arises from their multiple Fourier components. In this paper we demonstrate an efficient method to launch a self-collimated beam from a conventional waveguide, as well as a method to launch a particular Bloch mode from a conventional waveguide.
In Sections 2 and 3 we consider a silicon slab (refractive index $n_{\mathrm{Si}}=3.5$ ) of thickness $t=0.57 a$, where $a$ is the lattice parameter of the photonic crystal. This slab is patterned with a $2 \mathrm{D}$ square lattice of holes of radius $r=0.3 a$. The slab is surrounded by a material of index $n_{\text {cladding }}=1$ on both sides. In the photonic crystal literature, dimensions, frequencies, and $k$ vectors are usually given in normalized units. However, we chose a particular set of dimensions, so that the phenomena described below are valid for $\lambda=1.5 \mu \mathrm{m}$ and take place below the light line and that reported dimensions are realistic from a fabrication point of view ( $a=0.45 \mu \mathrm{m}, r=0.135 \mu \mathrm{m}$, $t=0.2565 \mu \mathrm{m}$, where $a$ is the lattice period, $r$ is the hole radius, and $t$ is the thickness of the slab). Furthermore $\lambda=1.5 \mu \mathrm{m}$ corresponds to Bloch modes in the second band of the photonic crystal, where equifrequency contours are approximate squares, so that self-collimation takes place at this wavelength. The PPC and its selfcollimation properties have been described extensively elsewhere. ${ }^{17}$

A silicon waveguide is placed above the photonic crystal with a $200-\mathrm{nm}$ vertical gap. In Section 2 the waveguide is oriented in the $\Gamma X$ direction, whereas in Section 3 it is aligned in the $\Gamma M$ direction. The cross section of the waveguide varies in the different cases.

\section{LAUNCHING OF A SELF-COLLIMATED BEAM}

In this section we locate a silicon waveguide of width $=350 \mathrm{~nm}$ and height $=100 \mathrm{~nm}$ above the PPC, with a spacing of $200 \mathrm{~nm}$. The waveguide is oriented in the $\Gamma X$ direction of the photonic crystal. This geometry is illustrated in Fig. 1.

The dimensions of the waveguide have been chosen so that the dispersion diagram of the stand-alone waveguide crosses the second band of the unperturbed PPC at 


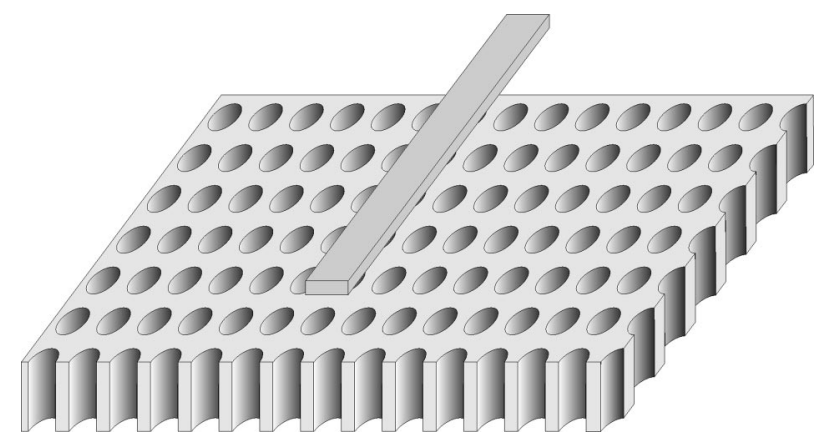

Fig. 1. Schematic view of a PPC and a waveguide oriented in the $\Gamma X$ direction above the photonic crystal.

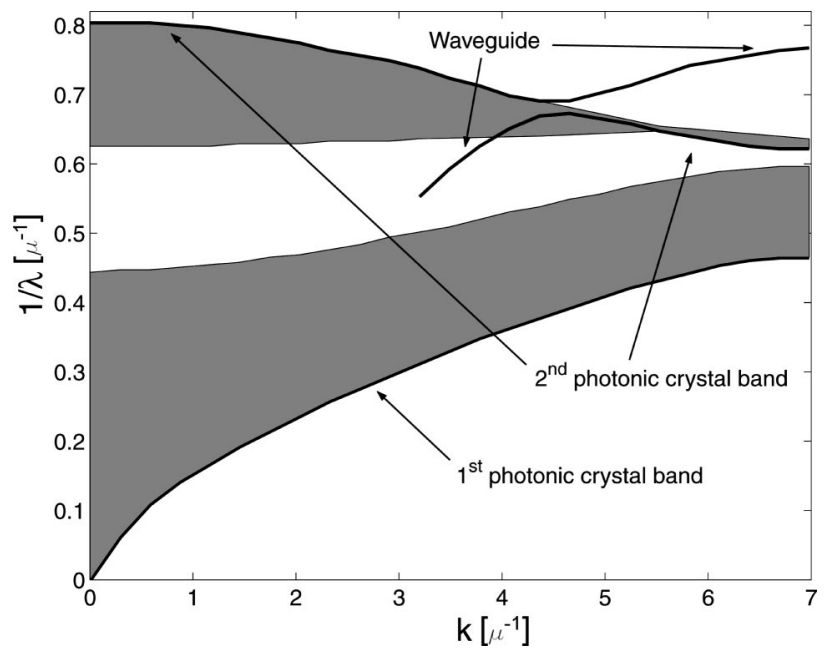

Fig. 2. Band diagram for the coupled PPC waveguide system. The $x$ axis gives the $k$-vector component imposed by the Bloch boundary condition in the $\Gamma X$ direction, and the $y$ axis gives the inverse wavelength $1 / \lambda=f / c$ of the resulting modes (where $f$ is the frequency and $c$ is the speed of light in vacuum). Labels indicate modes that correspond to the photonic crystal and to the waveguide far from the coupling region. The second band of the photonic crystal anticrosses with the waveguide. The dispersion diagram of the waveguide ends abruptly because the lowerfrequency modes were not resolved with the numerical method used (badly confined modes).

$\lambda=1.5 \mu \mathrm{m}$. The corresponding mode in the PPC is in the second band (folded back once). This has the crucial consequence that the PPC mode propagates in the opposite direction from the waveguide mode, since its group velocity $\left(v_{g}\right)$ points inside the equifrequency contour. Because two counterpropagating waves are coupled together, anticrossing occurs, and a mini stop band is opened in the coupled system (Fig. 2). Of course, the mini stop band is seen by the waveguide and the PPC only in the immediate vicinity of the waveguide. The PPC far from the waveguide remains unperturbed and has no stop band. The band diagram was obtained through finite-difference time-domain ${ }^{18}$ (FDTD) calculations by applying Bloch boundary conditions in the translation direction of the waveguide and Mur absorbing boundary conditions on the other boundaries. Discretization was chosen to be $10 \mathrm{~nm}$. In Fig. 2 the photonic crystal modes corresponding to the $\Gamma X$ direction and the waveguide modes are plotted as thick curves. Photonic crystal modes that are in arbitrary directions are not filtered out because Bloch boundary conditions are applied only in one direction. The intermediate gray zone results from those modes.

If light in the frequency range of the mini stop band is launched in the waveguide, it will exponentially decay in the coupled system. The light progressively couples into the PPC and backpropagates (Fig. 3). If now the waveguide starts where the pulse was launched (for example, if it is connected to a grating coupler), the light will propagate unperturbed in the photonic crystal behind the entry point, in the opposite direction from which it was originally launched and form a self-collimated beam. We performed a FDTD simulation at $\lambda=1.48 \mu \mathrm{m}$ (lower edge of the stop band) with a $20-\mathrm{nm}$ discretization to demonstrate this. Figure 3 shows two top views of the device and the out-of-plane component of the $B$ field, on the $x y$ symmetry plane of the waveguide [Fig. 3(a)] and on the $x y$-symmetry plane of the PPC [Fig. 3(b)]. It can clearly be seen that the field decreases exponentially in the $y$ direction and forms a self-collimated beam in the PPC.

Figure 4 shows how the field couples from the waveguide to the PPC. The field in the PPC seems to have a different $k_{y}$ from the field in the waveguide. This is because the waveguide couples to the Fourier component of the PPC Bloch mode that is in the first Brillouin zone, representing only a small fraction of the power contained within the mode. ${ }^{17}$ The apparent periodicity is dominated by the component in the second Brillouin zone, which is the dominant component of the Bloch mode.

To use this power-transfer mechanism in a device, it would be beneficial to have the ability to switch on and off

\begin{tabular}{|c|c|}
\hline \begin{tabular}{cccc|cccc}
0 & 0 & 0 & 0 & 0 & 0 & 0 & 0 \\
0 & 0 & 0 & 0 & 0 & 0 & 0 & 0 \\
0 & 0 & 0 & 0 & 0 & 0 & 0 & 0 \\
0 & 0 & 0 & 0 & 0 & 0 & 0 & 0 \\
0 & 0 & 0 & 0 & 0 & 0 & 0 & 0 \\
0 & 0 & 0 & 0 & 0 & 0 & 0 & 0 \\
0 & 0 & 0 & 0 & 0 & 0 & 0 & 0 \\
0 & 0 & 0 & 0 & 0 & 0 & 0 & 0 \\
0 & 0 & 0 & 0 & 0 & 0 & 0 & 0 \\
0 & 0 & 0 & 0 & 0 & 0 & 0 & 0 \\
0 & 0 & 0 & 0 & 0 & 0 & 0 & 0 \\
0 & 0 & 0 & 0 & 0 & 0 & 0 & 0 \\
0 & 0 & 0 & 0 & 0 & 0 & 0 & 0 \\
0 & 0 & 0 & 0 & 0 & 0 & 0 & 0 \\
0 & 0 & 0 & 0 & 0 & 0 & 0 & 0 \\
0 & 0 & 0 & 0 & 0 & 0 & 0 & 0 \\
0 & 0 & 0 & 0 & 0 & 0 & 0 & 0 \\
0 & 0 & 0 & 0 & 0 & 0 & 0 & 0 \\
0 & 0 & 0 & 0 & 0 & 0 & 0 & 0 \\
0 & 0 & 0 & 0 & 0 & 0 & 0 & 0 \\
0 & 0 & 0 & 0 & 0 & 0 & 0 & 0 \\
0 & 0 & 0 & 0 & 0 & 0 & 0 & 0 \\
0 & 0 & 0 & 0 & 0 & 0 & 0 & 0 \\
0 & 0 & 0 & 0 & 0 & 0 & 0 & 0 \\
0 & 0 & 0 & 0 & 0 & 0 & 0 & 0 \\
0 & 0 & 0 & 0 & 0 & 0 & 0 & 0 \\
0 & 0 & 0 & 0 & 0 & 0 & 0 & 0 \\
0 & 0 & 0 & 0 & 0 & 0 & 0 & 0
\end{tabular} & 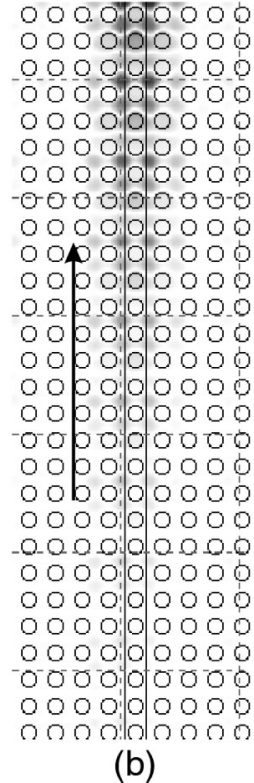 \\
\hline
\end{tabular}

Fig. 3. FDTD simulation of a decaying field in the mini stop band for a discretization of $20 \mathrm{~nm}$ and $\lambda=1.48 \mu \mathrm{m}$. The mode of the stand-alone waveguide is launched into the waveguide. (a) $B_{z}$ in the $x y$-symmetry plane of the waveguide, (b) $B_{z}$ in the $x y$-symmetry plane of the PPC. The decay of the coupled field is clearly seen. The field in the PPC propagates in the opposite direction from the field in the waveguide. 


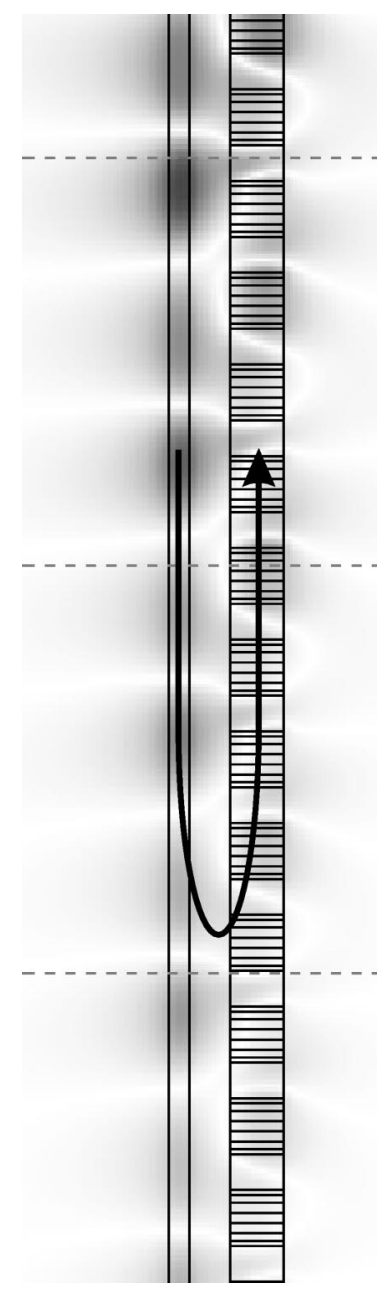

Fig. 4. FDTD simulation of a decaying field in the mini stop band for a discretization of $20 \mathrm{~nm}$ and $\lambda=1.48 \mu \mathrm{m}$. The mode of the stand-alone waveguide is launched into the waveguide. The figure shows $B_{z}$ in the $y z$-symmetry plane of the coupled structure. The field couples into the PPC and propagates in the opposite direction.

the coupling between the photonic crystal and the waveguide. To this effect, we introduce a second type of photonic crystal lattice, rotated by $45^{\circ}$ and rescaled by $1 / \sqrt{2}$, which we refer to as PC2. We refer to the original lattice as PC1. In a previous publication ${ }^{17}$ we showed that PC2 supports self-collimated beams, that a self-collimated beam can be transmitted from PC2 to PC1, and that the corresponding modes in PC2 lack the Fourier component in the first Brillouin zone of PC1 that is coupled to the waveguide (Fig. 5). Thus no anticrossing will occur if the waveguide is positioned above PC2. By substituting PC2 with PC1, we can switch the coupling on. The power is then transferred into a self-collimated beam in PC1 that backpropagates and crosses again the boundary between PC1 and PC2 (Fig. 6).

The coupling scheme proposed in this section is very similar to a counterdirectional coupler, ${ }^{19}$ in which two waveguides are coupled through the modulation of one or both of the waveguides, but in which the wave of waveguide 1 is coupled to a counterpropagating wave of wave- guide 2. In a directional coupler, full power transfer is achieved only through careful control of the coupling length. However, in the case of a counterdirectional coupler, the coupling efficiency is very robust to coupling length. This is of particular advantage with photonic crystals in which a small process bias on the hole size could severely affect the effective coupling length and thus the efficiency of a forward-coupling scheme.

In a counterdirectional coupler the coupling condition is given by $\mathbf{k}_{1}-\mathbf{k}_{2}=m \mathbf{K}$, where $m$ is an integer and $\mathbf{K}$ is the reciprocal lattice vector given by the periodicity of the waveguide modulation $(K=2 \pi / l$, where $l$ is the modulation period). This coupled-wave approach is intuitive in the small-perturbation limit. However, in the case in which a photonic crystal is coupled to a waveguide, the modulation is provided by the lattice of holes (high index contrast), which no longer satisfies smallperturbation assumptions. Coupled-mode theory must therefore be modified to take into account the deep grating. One approach is then to consider the coupling between the waveguide mode and the PPC Bloch mode rather than between counterpropagating waveguide modes. ${ }^{20}$ The periodic modulation of the medium is already taken into account by the structure of the Bloch mode, in which the Bloch mode has several $k$-space components. The component in the first Brillouin zone is the one that is coupled to the waveguide (at the crossing point in the reduced diagram in the first Brillouin zone, the waveguide has not been folded back; see Fig. 2). In the limit of vanishing modulation, the Bloch-mode component in the second Brillouin zone is the only one remaining, and coupling disappears.

There is one difference between our PPC coupler and a standard counterdirectional coupler. In a counterdirectional coupler, two waveguides are coupled together, whereas here a waveguide is coupled to a $2 \mathrm{D}$ system. This is important because, in a counterdirectional coupler, the field coupled from guide 1 to guide 2 and then back to guide 1 interferes destructively with what is left in guide 1. If a waveguide were to be coupled to a $2 \mathrm{D}$ slab with a one-dimensional modulation only in the direction of the waveguide, the field coupled in the slab would diffract, and only a small portion would couple back (this case can be described by a leaky waveguide). However, we choose the photonic crystal so that it supports selfcollimated beams, and, because of the orientation of the waveguide, it couples to self-collimated modes that remain collimated below the waveguide.

Optimum self-collimation is achieved above the light line for the photonic crystal described above. ${ }^{17}$ The above device was designed so that the anticrossing occurs just below the light line, so that the excited Bloch modes are lossless, with the strongest possible self-collimation effect. A similar design could be made for a silicon-silica material system. We have verified that a silica-clad square-lattice photonic crystal of dimensions $t=0.340$ $\mu \mathrm{m}, \quad a=0.5 \mu \mathrm{m}$, and $r=0.15 \mu \mathrm{m}$ supports selfcollimation in the second band at the wavelength $\lambda=1.52 \mu \mathrm{m}$. However those modes are above the light line in silica and are leaky, limiting the practical use of such a structure. In a silicon-silica material system, it 

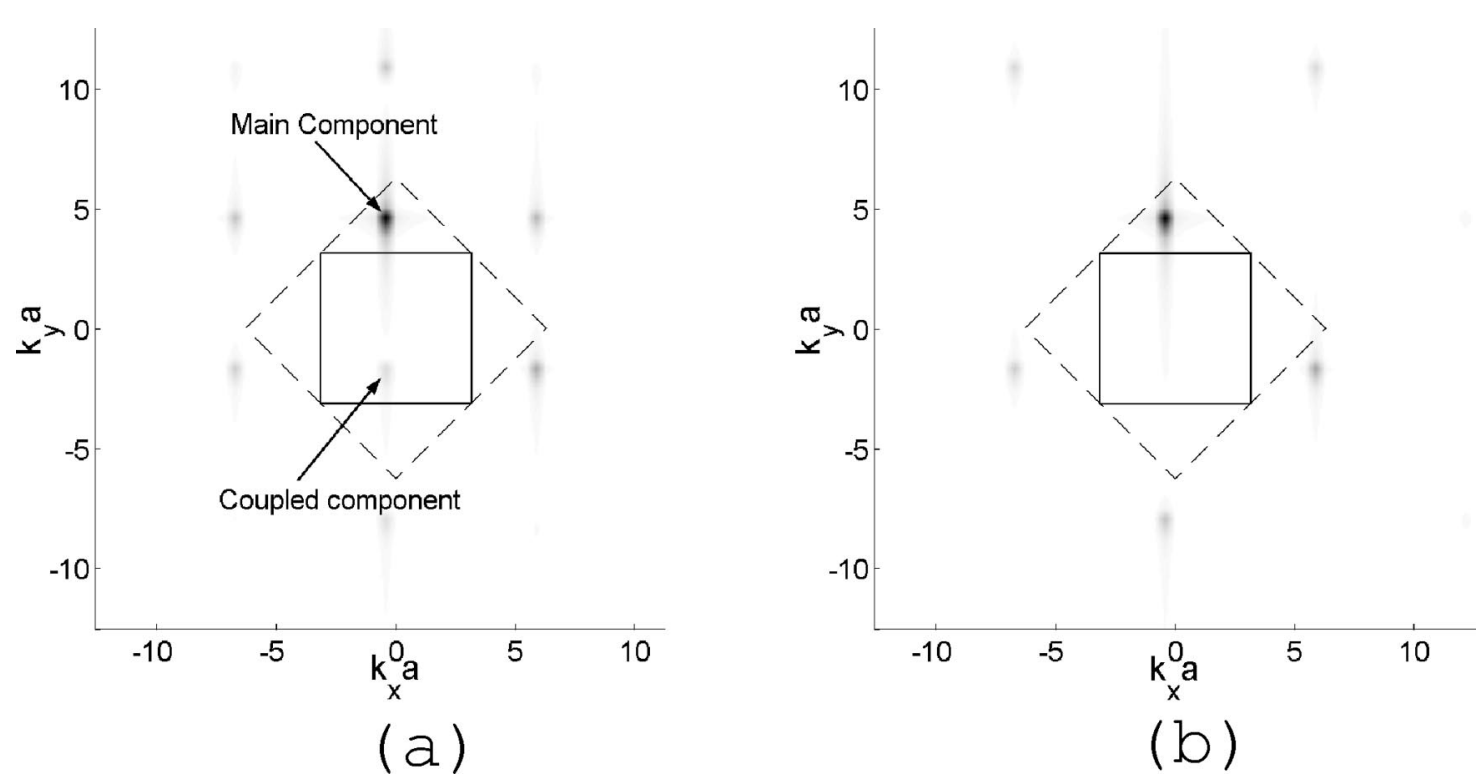

Fig. 5. (a) Spatial Fourier transform of a typical Bloch mode of the second band of PC1 and (b) the spatial Fourier transform of the corresponding Bloch mode (same frequency and same main component $k$ vector) of PC2. The first Brillouin zone of PC1 is indicated by the solid-line square, and the second Brillouin zone is indicated by the dashed-line square. The PC2 Bloch mode misses the component in the first Brillouin zone that is coupled to the waveguide. Thus, by transmission of a beam from PC1 to PC2, the coupling to the waveguide can be switched on and off. Note that, because PC2 is rescaled and rotated as compared with PC1, the first Brillouin zone of PC2 corresponds to both the first and the second Brillouin zones of PC1. This explains why a Bloch mode of PC2 has only one Fourier component, whereas a Bloch mode of PC1 has two. $k$-vector components are given in dimensionless units of $k \cdot a$, where $a$ is the lattice parameter of PC1.

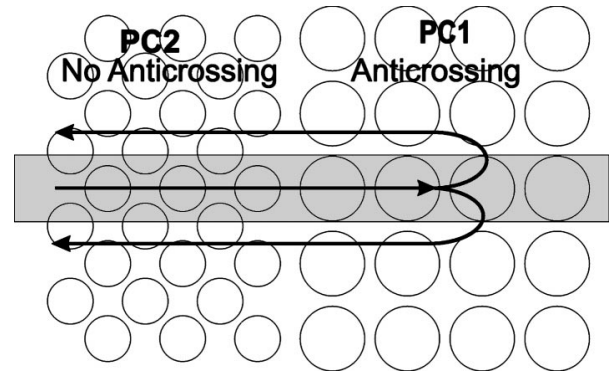

Fig. 6. A waveguide is situated above a photonic crystal with a transition between lattices PC2 and PC1. This way the anticrossing between the photonic crystal and the waveguide can be turned on and off, and a self-collimated beam can be launched into PC2.

would thus be advisable to use self-collimation in the first band (below the light line and lossless) in a forwardcoupling scheme.

\section{SELECTIVE EXCITATION OF A GIVEN BLOCH MODE}

If the waveguide is oriented in the $\Gamma M$ direction rather than the $\Gamma X$ direction, it is coupled to only a few Bloch modes, and, as we will show, it is possible to selectively excite a single Bloch mode. The Bloch modes excited in the photonic crystal have a propagation direction that is different from the waveguide direction, and therefore the coupled system behaves as a leaky waveguide. We again use FDTD simulation of such systems with 10-nm discretization and perform a spatial Fourier transform of $B_{z}$ in the center plane of the photonic crystal $(15 \times 15$ periods, or $675 \times 675$ computational points), so as to map the $\mathrm{TE}$ Bloch modes excited in the photonic crystal. It should be noted that TM modes are marginally excited, although the waveguide was excited with a TE mode. Because the $x y$-plane symmetry is broken in the coupled system, the overlap integral between the TE waveguide mode and the TM photonic crystal mode is nonzero.

We show the results for two waveguide geometries to emphasize methods for achieving single-mode excitation. In the first case, we took a waveguide of dimensions $520 \mathrm{~nm} \times 100 \mathrm{~nm}$, positioned above the PPC with a $200-n m$ vertical gap. When light couples into the photonic crystal, the $k$-vector component in the waveguide direction $\left[1 / \sqrt{(2)} \cdot\left(\mathbf{e}_{x}+\mathbf{e}_{y}\right)\right]$ is conserved modulo reciprocal lattice vectors of the photonic crystal (the coupled waveguide and photonic crystal system are still a periodic structure in this direction). This property discriminates the Bloch modes to which the waveguide can couple. Figure 7(a) illustrates this. The squarish contours are an idealized form of the equifrequency contours of the photonic crystal at $\lambda=1.5 \mu \mathrm{m}$, and the conserved $k$-vector component of the waveguide mode (component in the direction of the waveguide) is represented by an axis. Any PPC mode that has a nonvanishing Fourier component on that axis is coupled to the waveguide. It can be seen that only four photonic crystal modes are coupled to the waveguide. Figure 7(b) shows the propagation directions of those modes in real space. The propagation direction is given by the group velocity $\nabla_{k}(\omega)$, which is perpendicular to the equifrequency contour and points inside the equifrequency contour in the case of the second band. Although four modes are excited, there are only two modes on either side of the waveguide. To illustrate this, we performed a Fourier transform of the field on the left side and labeled each Fourier component with the corresponding Bloch mode (Fig. 8). 
To obtain single-mode excitation on each side of the waveguide, we changed the waveguide geometry to $400 \mathrm{~nm} \times 200 \mathrm{~nm}$. This waveguide has a larger effective index and a smaller $k$ vector, so that the situation shown in Fig. 9(a) is obtained. The waveguide was designed so that $k \cdot a=0.4$ was slightly smaller than $\sqrt{(2)} \cdot \pi$ $=4.44$, and thus the waveguide $k$ vector is slightly inside the first Brillouin zone, near the corner. It can be seen in

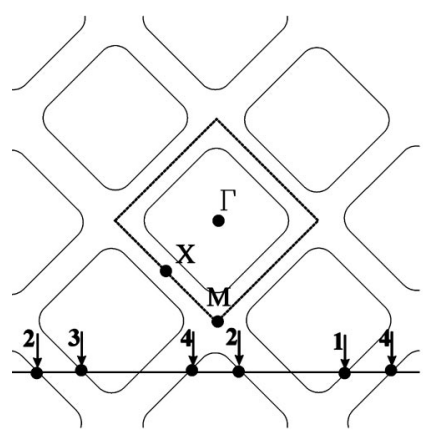

(a)

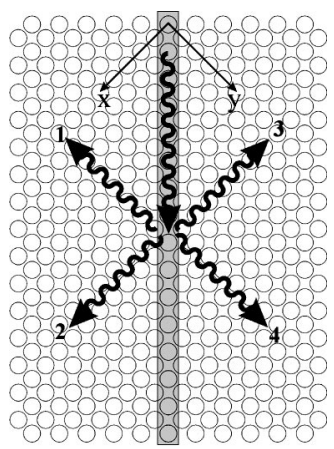

(b)
Fig. 7. Excited modes can be determined by conservation of the $k$-vector component in the $\Gamma M$ direction (modulo an inverse lattice vector). (a) The idealized equifrequency contour in the first Brillouin zone (dashed-line square), as well as its multiple higher-order copies. The horizontal line is determined by the $k$ vector of the waveguide mode and intersects all the PPC modes to which the waveguide couples. Four modes are excited, labeled 1 to 4 . The high-symmetry points are shown on the diagram $(\Gamma, M$, and $X)$. The directions of propagation (given by the group velocity) are indicated in (b) for each of the excited modes.

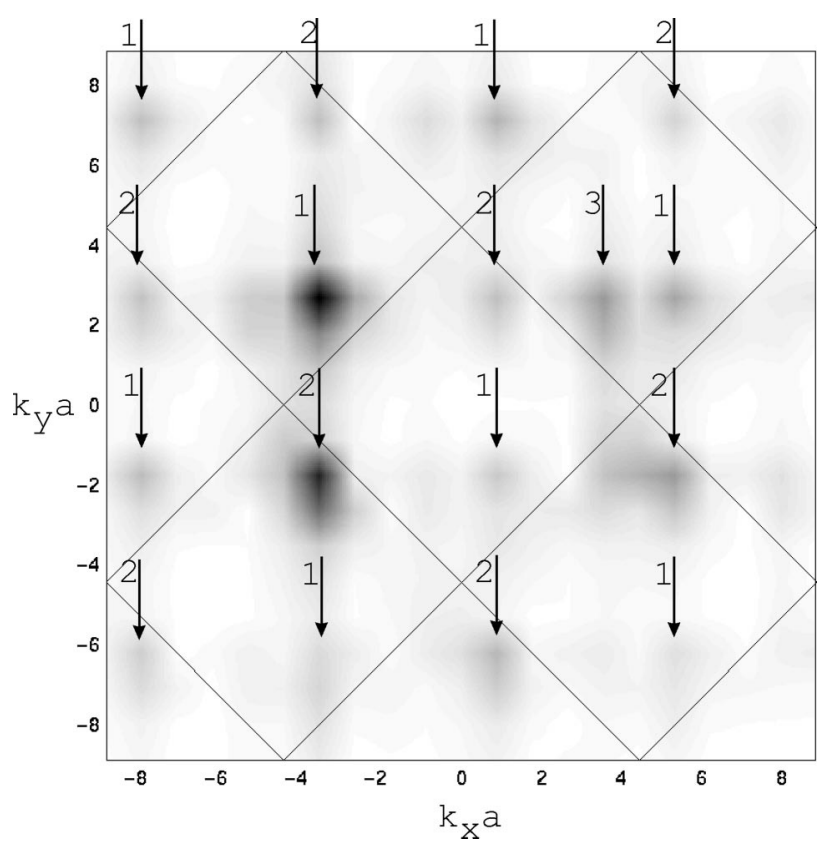

Fig. 8. Spatial Fourier transform of $B_{z}$ on the left side of the waveguide, on the center plane of the PPC $(z=0)$. Modes 1 and 2 that propagate to the left of the waveguide are present. The field was simulated by a FDTD with a $10-\mathrm{nm}$ discretization, waveguide dimensions are $520 \mathrm{~nm} \times 100 \mathrm{~nm}$, and $\lambda$ $=1.54 \mu \mathrm{m}$. The device is in the regime illustrated in Fig. 7 . The Fourier components are labeled in the same way as in Fig. 7. $k$-vector components are given in dimensionless units of $k \cdot a$, where $a$ is the lattice parameter.

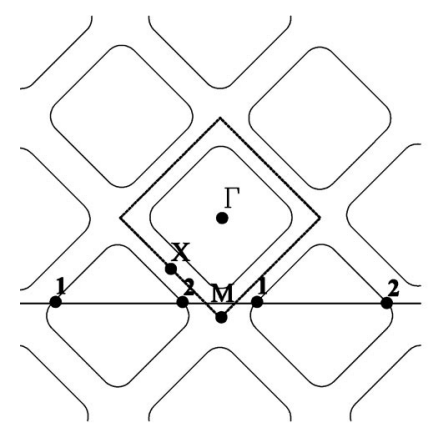

(a)

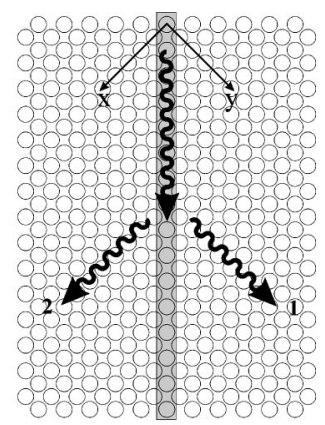

(b)
Fig. 9. Excited modes can be determined by conservation of the $k$-vector component in the $\Gamma M$ direction (modulo an inverse lattice vector). (a) The idealized equifrequency contour in the first Brillouin zone (dashed-line square), as well as its multiple higher-order copies. The horizontal line is determined by the $k$ vector of the waveguide mode and intersects all the PPC modes to which the waveguide couples. Two modes are excited, labeled 1 and 2 . The high-symmetry points are shown on the diagram $(\Gamma, M$ and $X$ ). The directions of propagation (given by the group velocity) are indicated in (b) for each of the excited modes.

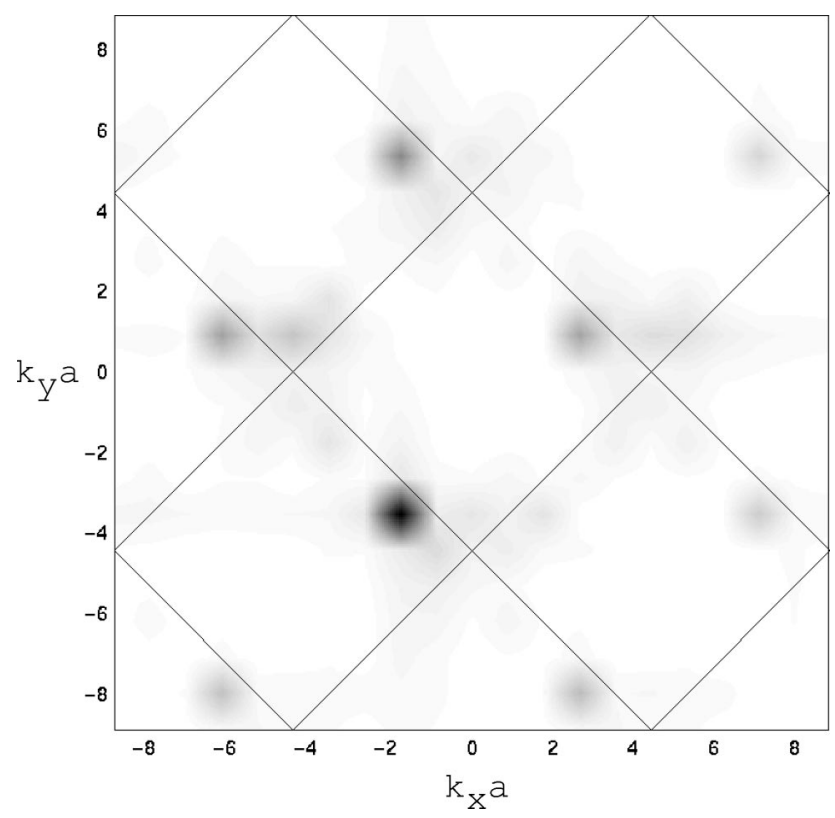

Fig. 10. Spatial Fourier transform of $B_{z}$ on the left side of the waveguide, on the center plane of the PPC $(z=0)$. The field was simulated by FDTD with a 10-nm discretization, waveguide dimensions are $400 \mathrm{~nm} \times 200 \mathrm{~nm}$, and $\lambda=1.54$. All the Fourier components belong to the same Bloch mode, and true singlemode excitation is achieved on either side of the waveguide. The device is in the same regime as that illustrated in Fig. 9. $k$-vector components are given in dimensionless units of $k \cdot a$, where $a$ is the lattice parameter.

Fig. 10 that all the Fourier components belong to the same Bloch mode.

There is only one Bloch mode excited on each side of the waveguide, but for a Lorentzian broadening around the central mode that is due to the decay length of light in the waveguide. We expect that by increasing the separation between the waveguide and the photonic crystal, a narrower mode distribution would be obtained, but we have not collected quantitative data on this. 


\section{CONCLUSION}

In conclusion we have shown that, through out-of-plane coupling between a planar photonic crystal and a waveguide located above the photonic crystal, a self-collimated beam, as well as a single Bloch mode, can be launched into the photonic crystal. This type of device permits the integration of self-collimation-based devices and conventional integrated optics.

\section{ACKNOWLEDGMENTS}

The authors acknowledge funding from the Defense Advanced Research Projects Agency under contract MDA 972-00-1-0019 and from the U.S. Army Research Office under contract DAAD 19-00-1-0392.

J. Witzens, the corresponding author, can be reached by e-mail atwitzens@caltech.edu.

\section{REFERENCES}

1. E. Yablonovitch, "Inhibited spontaneous emission in solidstate physics and electronics," Phys. Rev. Lett. 58, 2059 2062 (1987)

2. T. F. Krauss, R. M. De La Rue, and S. Brand, "Twodimensional photonic-bandgap structures operating at near infrared wavelengths," Nature 383, 699-702 (1996).

3. S. G. Johnson, S. H. Fan, P. R. Villeneuve, J. D. Joannopoulos, and L. A. Kolodziejski, "Guided modes in photonic crystal slabs," Phys. Rev. B 60, 5751-5758 (1999).

4. D. Labilloy, H. Benisty, C. Weisbuch, T. F. Krauss, R. M. De La Rue, V. Bardinal, R. Houdré, U. Oesterle, D. Cassagne, and C. Jouanin, "Quantitative measurement of transmission, reflection, and diffraction of two-dimensional photonic band gap structures at near-infrared wavelengths," Phys. Rev. Lett. 79, 4147-4150 (1997).

5. O. Painter, R. K. Lee, A. Yariv, A. Scherer, J. D. O'Brian, P. D. Dapkus, and I. Kim, "Two-dimensional photonic bandgap defect mode laser," Science 284, 1819-1821 (1999).

6. M. Lončar, D. Nedeljković, T. Doll, J. Vučković, A. Scherer, and T. P. Pearsall, "Waveguiding in planar photonic crystals," Appl. Phys. Lett. 77, 1937-1939 (2000).
7. C. J. M. Smith, R. M. De La Rue, M. Rattier, S. Olivier, H. Benisty, C. Weisbuch, T. F. Krauss, U. Oesterle, and R. Houdré, "Coupled guide and cavity in a two-dimensional photonic crystal," Appl. Phys. Lett. 78, 1487-1489 (2001).

8. S. Noda, A. Chutinan, and M. Imada, "Trapping and emission of photons by a single defect in a photonic bandgap structure," Nature 407, 608-610 (2000).

9. H. Kosaka, T. Kawashima, A. Tomita, T. Sato, and S. Kawakami, "Photonic-crystal spot-size converter," Appl. Phys. Lett. 76, 268-270 (2000).

10. P. Lalanne and A. Talneau, "Modal conversion with artificial materials for photonic-crystal waveguides," Opt. Express 19, 354-359 (2002); www.opticsexpress.org.

11. M. Boroditsky, R. Vrijen, T. F. Krauss, R. Coccioli, R. Bhat, and E. Yablonovitch, "Spontaneous emission extraction and Purcell enhancement from thin-film 2-D photonic crystals," J. Lightwave Technol. 17, 2096-2112 (1999).

12. V. N. Astratov, I. S. Culshaw, R. M. Stevenson, D. M. Whittaker, M. S. Skolnick, T. F. Krauss, and R. M. De La Rue, "Resonant coupling of near-infrared radiation to photonic band structure waveguides," J. Lightwave Technol. 17, 2050-2057 (1999).

13. Y. Xu, R. K. Lee, and A. Yariv, "Adiabatic coupling between conventional dielectric waveguides and waveguides with discrete translational symmetry," Opt. Lett. 25, 755-757 (2000).

14. A. Mekis and J. D. Joannopoulos, "Tapered couplers for efficient interfacing between dielectric and photonic crystal waveguides," J. Lightwave Technol. 19, 861-865 (2001).

15. H. Kosaka, T. Kawashima, A. Tomita, M. Notomi, T. Tamamura, T. Sato, and S. Kawakami, "Self-collimating phenomena in photonic crystals," Appl. Phys. Lett. 74, 1212-1214 (1999).

16. H. Kosaka, T. Kawashima, A. Tomita, M. Notomi, T. Tamamura, T. Sato, and S. Kawakami, "Superprism phenomena in photonic crystals," Phys. Rev. B 58, R10096-R10099 (1998).

17. J. Witzens, M. Lončar, and A. Scherer, "Self-collimation in planar photonic crystals,” IEEE J. Sel. Top. Quantum Electron. 8, 1246-1257 (2002).

18. A. Taflove, Computational Electrodynamics: The FiniteDifference Time-Domain Method (Artech House, Norwood, Mass., 1995).

19. K. Okamoto, Fundamentals of Optical Waveguides (Academic, NTT Photonics Laboratories, Japan, 2000).

20. C. M. de Sterke, D. Salinas, and J. E. Sipe, "Coupled-mode theory for light propagation through deep nonlinear gratings," Phys. Rev. E 54, 1969-1989 (1996). 